\title{
CELESTIAL OPTICAL TRANSIENTS FROM 532 BCE TO 2015 AD. FROM THE RIDICULOUS TO THE SUBLIME?
}

\section{Brian Warner*}

Astrophysics, Cosmology and Gravity Centre, Department of Astronomy, University of Cape Town

E-mail: brian.warnereuct.ac.za

\begin{abstract}
Three, and now a probable fourth, novae that were observed by oriental astrologers more than a thousand years ago have been identified in recent years as dwarf novae. This adds strength to the theory that the long-term evolution of most cataclysmic variable stars consists of continuous episodes as nova-likes, dwarf novae, or in hibernation, interrupted by occasional (and rare) nova eruptions.
\end{abstract}

$3 r d$ Annual Conference on High Energy Astrophysics in Southern Africa-HEASA2015,

18-20 June 2015

University of Johannesburg, Auckland Park, South Africa

\footnotetext{
*Speaker.
} 


\section{Introduction}

The earliest recorded optical transients occur in oriental records at least as early as $532 \mathrm{BCE}$ (there is a possible earlier record of a nova, c.1400 BCE, on Chinese oracle bones (Hsi 1958), but with insufficient detail to be of modern value). Among these are events now recognised as novae and supernovae, the most famous of which is the supernova of AD1054, with the Crab Nebula as remnant. Within the past few years some of the ancient ordinary novae have been identified from their ejected shells or from the nature of the remnant binary star; more can be expected to be found with the upcoming all-sky surveys, such as LSST and Pan-STARRS. Here I discuss the current status of the observations and the recent discovery of an eclipsing dwarf nova, with an ejected shell, that is probably the remnant of the Chinese nova of AD 483.

\section{Oriental Transients}

Records were kept by Chinese, Japanese and Korean imperial astrologers who had been instructed to keep their emperors informed on the state of the heavens, which were considered to reflect the state of terrestrial affairs. Included in the records inter alia were new (guest) stars, rainbows, sunspots, snowstorms, comets, meteors, meteor showers, solar and lunar eclipses, aurorae, lightning, and hail. Among the new stars were what we now recognise as novae and supernovae, including the supernova of 4 July AD 1054, only identified for certain as the origin of the Crab Nebula by Oort in 1942. There are several catalogues that list and interpret the oriental records, the most important of which are Hsi (1958), Ho (1962), Clark \& Stephenson (1977), Xu, Pankanier \& Jiang, (2000), Nickiforov (2010).

A complication that arises in relating oriental guest stars to modern objects is the absence of an early universal grid system, like declination and right ascension. Instead, a 'mansion' or 'palace' reference was used, defined by a bright asterism and the strip of sky from pole to horizon that included that asterism at the time of observation. There were 28 mansions, mostly near the ecliptic plane (to describe where the moon was at any time); locations of novae deduced from the oriental descriptions are rarely better than about 10 degrees (Nickiforov 2010).

\section{Modern Identification}

Current understanding of the evolution of cataclysmic variable (CV) stars is that they are all close binary stars, with mass transferring from (usually) a main sequence donor onto a white dwarf (Warner 1995). The build-up of hydrogen-rich material on the surface of the white dwarf leads eventually to a thermonuclear runaway, with expulsion of the accreted gas; intervals between such nova eruptions in theory should be $\sim 10^{4-5} \mathrm{y}$. Following the eruption, the heated white dwarf cools down, reducing irradiation of the donor and hence the mass transfer rate (perhaps to zero for a long time - leading to a phase of "hibernation" (Shara et al 1986)); during the descent or subsequent rise the star becomes a standard dwarf nova, with cyclical outbursts of its accretion disc - an example is GK Per, which was nova Per in 1901 and started outbursts around 1966 (brightening $\sim 3$ magnitudes every $\sim 3 \mathrm{y}$ ). 


\title{
Estimate of the Chinese astronomy development through the descriptions of nova observations
}

\author{
Mihael G. Nickiforov \\ Moscow State University, Russia \\ michael.nickiforov@gmail.com \\ (Research report. Accepted on 19.05.2009)
}

\begin{abstract}
More than a hundred descriptions of guest stars observations made by Chinese Korean and Japanese astronomers from 6th till 17th centuries are examined in this work. The comparison of the distribution of star outbursts on galactic latitudes from the Chinese sources with the modern data casts doubt on the reliability of the ancient observations. The text analysis shows that the technique of the descriptions of the places of outbursts of new stars does not change from the first ancient observations till the 16th century. This is a sign of a lack of precise instrumental measurements until the arrival of the Jesuits, in 16th century, and of a lack of Chinese star catalog in the medieval China.

Key words: history of astronomy, nova stars, guest stars
\end{abstract}

Appendix. List of nova stars.

\begin{tabular}{|c|c|c|c|c|c|c|}
\hline N & Date & Asia & Europe & $\mathbf{l}_{\text {gal }}$ & $\mathbf{b}_{\text {gal }}$ & Comment \\
\hline N1 & spring, -532 & LM10 & $\epsilon$ Aqr & 38 & -30 & China \\
\hline N2 & $14.08 \div 12.09 .-204$ & Dajiao & $\alpha$ Boo & 15 & 69 & China, 10 days; fuzzy \\
\hline N3 & $23.06 \div 21.07 .-134$ & LM4 & $\pi$ Sco & 347 & 20 & China \\
\hline N4 & $-110 \div-105$ & Heshu & $\beta$ Gem & 192 & 23 & China \\
\hline N5 & $-104 \div-101$ & Zhaoyao & $? ?$ & - & - & China, fuzzy \\
\hline N6 & $17.10 \div 15.11 .-77$ & Zigong & $\epsilon$ UMi & 115 & 31 & Chi; between $\alpha, \beta$ UMi \\
\hline N7 & $03.05 \div 31.05 .-48$ & LM8 & $\varphi$ Sgr & 8 & -11 & China; bluish-white \\
\hline N8 & $20.06 \div 18.07 .-47$ & Juanshe & $\mu$ Per & 154 & -10 & China \\
\hline N9 & $24.04 .-4$ & Hegu & $\alpha$ Aql & 48 & -9 & China \\
\hline N10 & $? . ? .29$ & Yuzuo & $\beta$ UMi & 113 & 41 & China; near Yuzuo \\
\hline
\end{tabular}

Figure 1: Extract from Nickiforov, M. G., 2010, Bulgarian AJ, 13, 116, showing the title and abstract as well as part of the appendix.

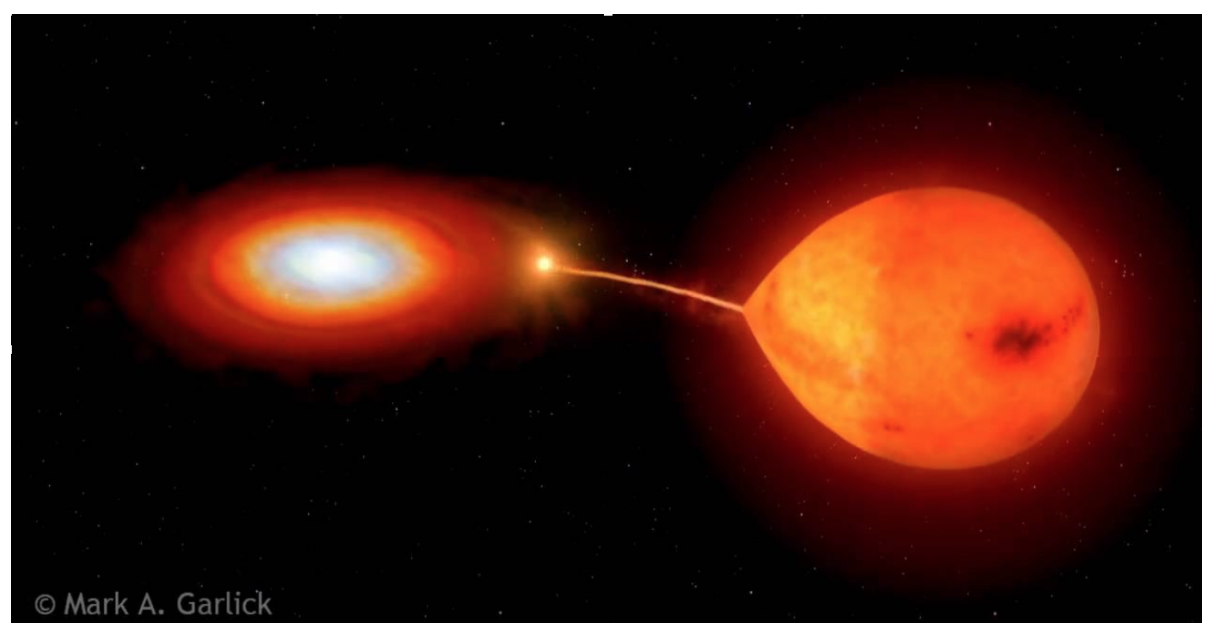

Figure 2: Artist's impression of a CV, copyright: Mark A. Garlick. 


\subsection{BK Lyn}

BK Lyn was identified in 1982 in a search for stars with ultraviolet excesses and later found from spectra to be a CV with orbital period 108 mins and very low amplitude photometric variations. Spectroscopically it was classified as a nova-like variable, the only one with a period less than $2 \mathrm{~h}$. Assiduous monitoring for 20 years paid off during 2011 when BK Lyn transformed into a dwarf nova, with outburst intervals $\sim 5 \mathrm{~d}$ (Patterson et al 2013). Hertzog (1986) proposed from a study of the oriental novae that BK Lyn can be securely identified as the remnant of Nova Lyn AD 101 (N15 in Nickiforov's (2010) list; positioned very accurately relative to a bright star (Hsi 1958)).

\subsection{Z Cam}

$\mathrm{Z}$ Cam is the type star of dwarf novae that have standstills and is one of the brightest and best observed CVs. Not until 2007 was it noticed that the GALEX (ultraviolet) image of Z Cam is partly surrounded by an emission nebulosity with a radius of $\sim 15^{\prime}$ (Shara et al 2007, 2012a), physically an order of magnitude larger than the size of novae observed during the past century. The derived mass of the shell is similar to that of recent novae, and is much larger than what would be ejected by winds during dwarf nova outbursts. There is no detectable expansion of the shell, which is presumed to have been decelerated by interaction with the interstellar medium.

Johansson (2007) pointed out that a guest star in 77 BCE located between $\alpha$ UMi (the North Pole star) and $\alpha \mathrm{UMa}$ could be the origin of the nova shell. I note, however, that there are possible alternative identifications with novae near $\alpha$ UMi in AD 158 and AD 305 (Chinese novae N19 and N28 in Nickiforov's (2010) list).

\subsection{AT Cne}

Shara et al (2012b) have found a second fragmented nova shell around a Z Cam star - that of AT Cnc. It is $3^{\prime}$ in diameter and has an ejecta mass $\sim 5 \times 10^{-5} M_{\odot}$. AT Cnc has galactic coordinates $\left(198^{\circ}, 32^{\circ}\right)$ which are close to the Korean novae of $1031 \mathrm{AD}\left(207^{\circ}, 30^{\circ}\right)$ and $1645 \mathrm{AD}\left(207^{\circ}, 30^{\circ}\right)$ in Nickiforov's (2010) list.

\subsection{Te 11}

Jacoby et al (2010) reported on a professional/amateur (largely citizen science) project that searched for previously unknown planetary nebulae (PN) recorded on digital sky surveys. Among the $\sim 60 \mathrm{PN}$ candidates one stood out as having unusual morphology and spectrum; Te11 has a low excitation nebula spectrum, is near to the large gaseous structure known as Barnard's Loop and a distorted shape which was noted to be close to the molecular cloud complex that surrounds (in projection) the stars in Orion's Belt. Much of this Orion emission nebulosity appears close to $\zeta$ Ori at a distance $\sim 400 \mathrm{pc}$ (Goudis 2012), which is very close in the sky (and in distance - see later) to Te11.

In their ongoing photometric study of faint CVs, especially outbursting dwarf novae, Woudt and Warner observed the Catalina Real-Time Transit Survey (Drake et al 2009) source

CSS111003:054558+022106 during an outburst in December 2013, finding it to be an eclipsing 


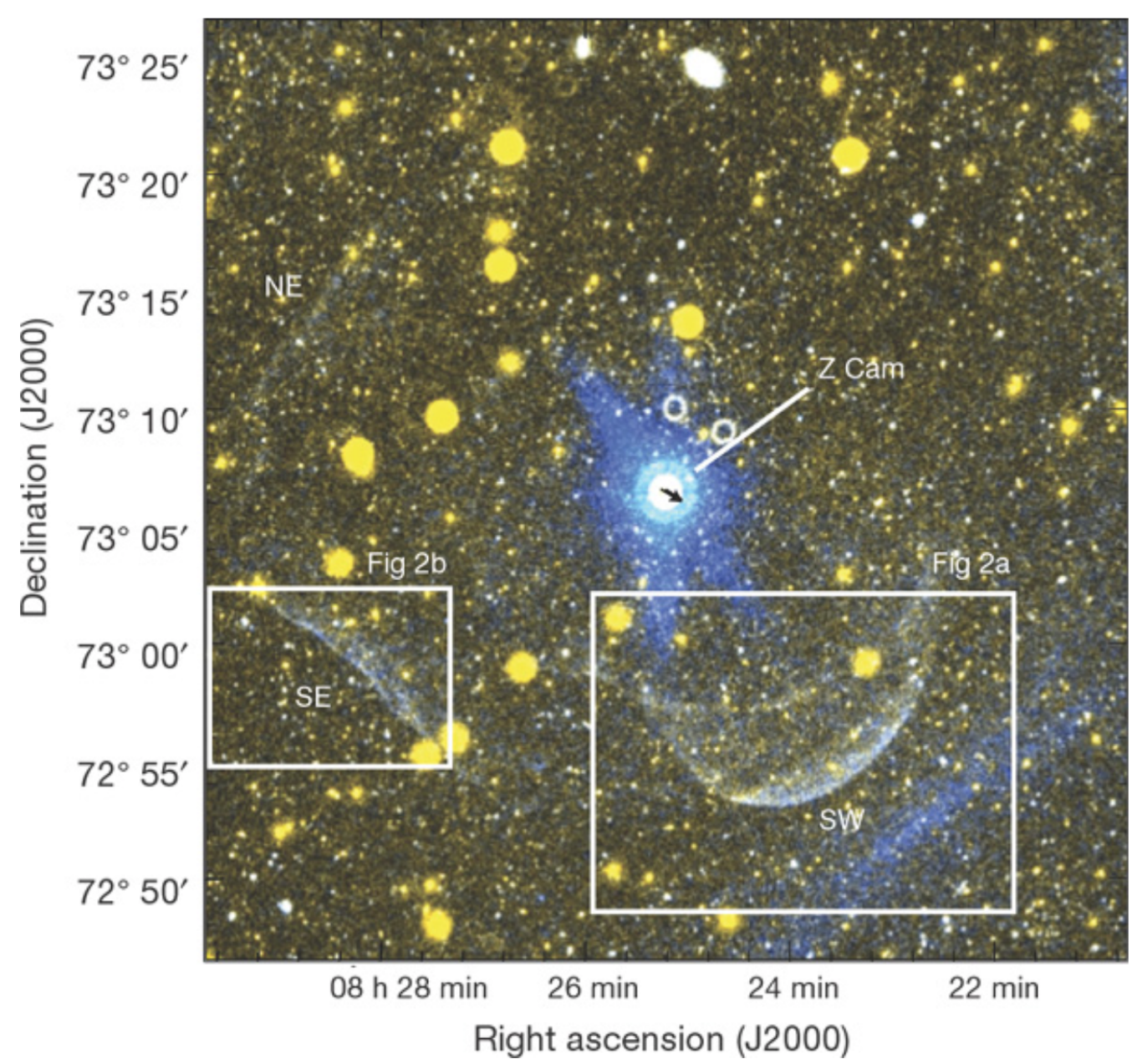

Figure 3: Figure from Shara et al (2007) highlighting the emission nebulosity around Z Cam.

system with orbital period of $2.90 \mathrm{~h}$ (just within the CV orbital period gap). The CRTS light curve shows 5 recorded outbursts over 10 years.

Independently, in his follow-up survey of planetary nebulae Brent Miszalski had photometrically observed the central star of Te11 and found it to have eclipses.

Comparisons of observations showed that Te11 and the CSS star are identical - the central star of Te11 is a classic dwarf nova. This excludes it from being a planetary nebula, the central stars of which are hot subdwarfs, and their nebulae are of high excitation. Further photometric and spectroscopic observations, and modelling (Miszalski et al 2015), of Te11 have shown that its white dwarf has a mass $\sim 1.2 M_{\odot}$ and effective temperature $\sim 13000 \mathrm{~K}$. The high mass suggests that there should be nova eruptions more frequently than those of classical novae (i.e. a Recurrent Nova, with eruptions every hundreds or thousands of years: Warner 1995). Using the relationship given by Warner (1987) and recalibrated by Patterson (2013) between orbital period, disc inclination and maximum apparent brightness during dwarf nova outbursts, together with modelling of the dwarf 


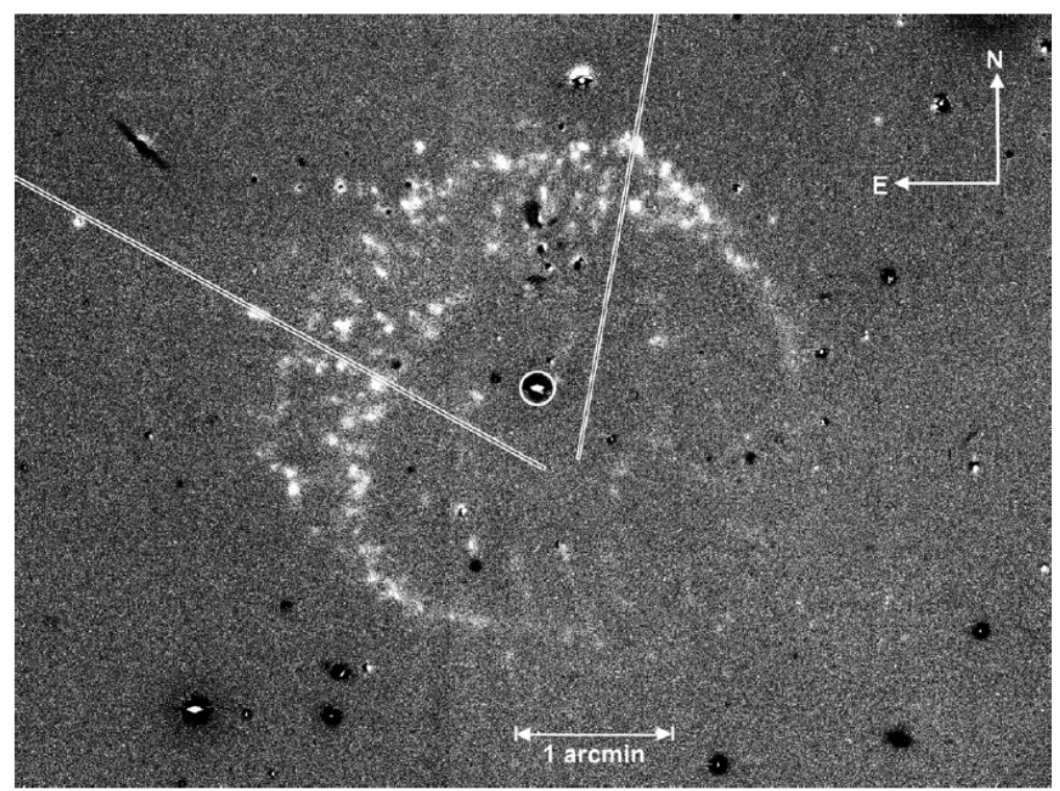

Figure 2. Net $\mathrm{H} \alpha+[\mathrm{N}$ II] image of AT Cnc. AT Cnc is circled. The positions of two slits used to obtain spectra of the AT Cnc ejecta are superposed on the image.

Figure 4: Figure from Shara et al (2012b) where a second fragmented nova shell is clearly seen around AT Cnc.

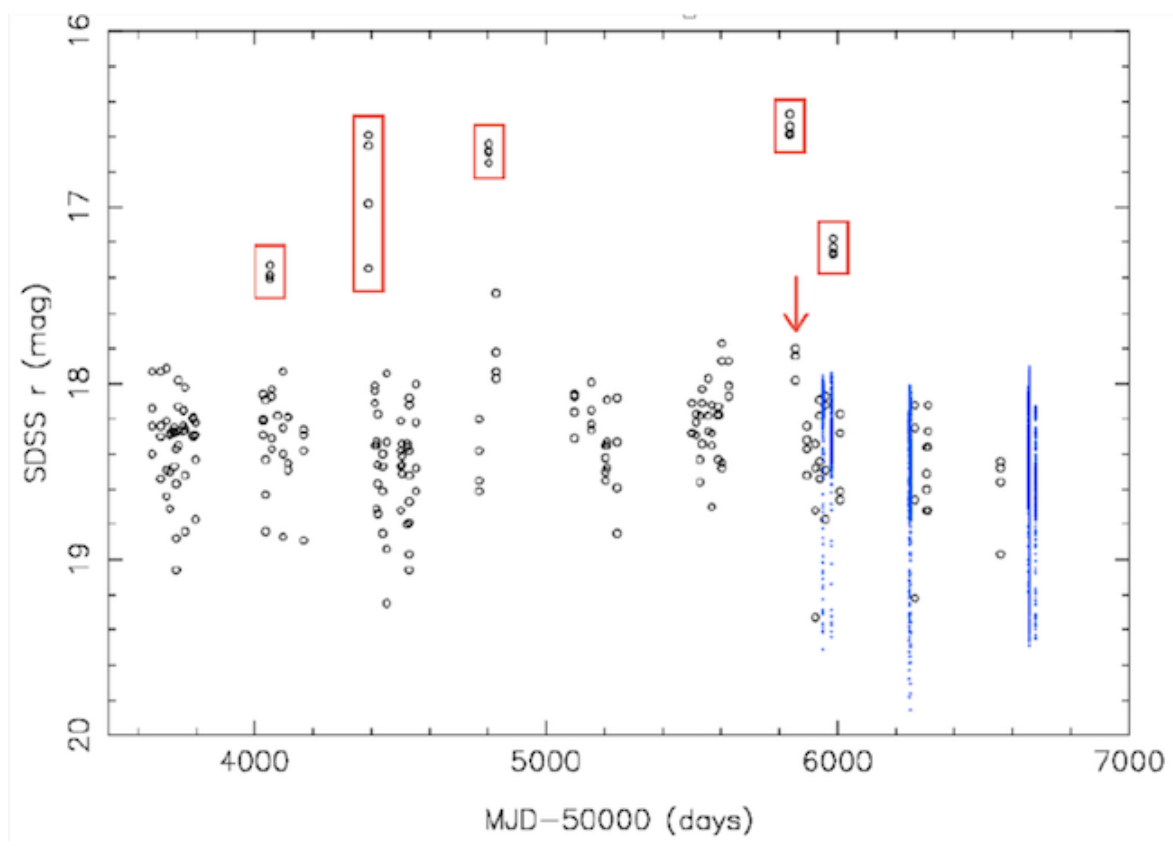

Figure 5: Figure from Drake et al (2009) highlighting the outbursts and eclipses of Te 11. 
nova quiescent light curve, give a distance to the star of $330 \pm 50 \mathrm{pc}$.

The gas in the Orion OB Association is mostly at a distance of $460 \mathrm{pc}$ (Goudis 1982), including Barnard's Loop, which Te11 is close to and may be immersed in.

\section{Te11 as the remnant of Nova Ori AD 483}

The location of Te11 just to the east of the Orion's Belt star $\zeta$ Ori (Alnitak in early European astronomy) shortens any search for an early oriental candidate. $\zeta$ Ori is a significant star in ancient Chinese astronomy - it is the dominant star in Lunar Mansion 21 (Shen, or Three Stars in translation - referring to Orion's Belt). There is only one obvious candidate: Nova N40 in Nickiforov's (2010) list - the nova of 14 December AD 483, which was described as "Emperor Xisosen of Wei, 7th year of the Taihe reign, period 10th month. There was a guest star east of Shen as large as a peck measure and like a fuzzy star". The reference to "fuzzy star" might be thought to indicate a comet, but Nickiforov (2010) finds that many of the "fuzzy star" descriptions cannot refer to comets and must be novae.

The suggested nova remnant around Te11 is relatively small, which may be the result of rapid deceleration of the expanding nova ejecta by the gas in the Orion region (10 or 100 times the density of the ISM). One side of the remnant appears to be a shock caused by ploughing of the ejecta into the surrounding gas.

\section{Conclusion}

With additional identifications of remnants from early oriental novae it should be possible to map out the longer-term development of ejecta shells and the recovery of their central stars as normal cataclysmic variable between eruptions.

\section{Acknowledgments}

Details of the Te11 observations will be published separately (Miszalski et al, 2015), the part given here is largely that contributed by myself. I am grateful to Brent Miszalski and Patrick Woudt for provision of some of the illustrations, and to Kerry Paterson for assistance with layout of the text. My research is supported by the National Research Foundation and by the University of Cape Town.

\section{References}

[1] Clark, D.H., Stephenson, F.R., 1977, The Historical Supernovae, Pergamon Press, New York.

[2] Drake, A. J. et al, 2009, ApJ 696, 870.

[3] Goudis, C. D., 1982, The Orion Complex, A Case Study of Interstellar Matter, ApSpLibr, Vol. 90.

[4] Hertzog, K. P., 1986. Observatory, 106, 38. 


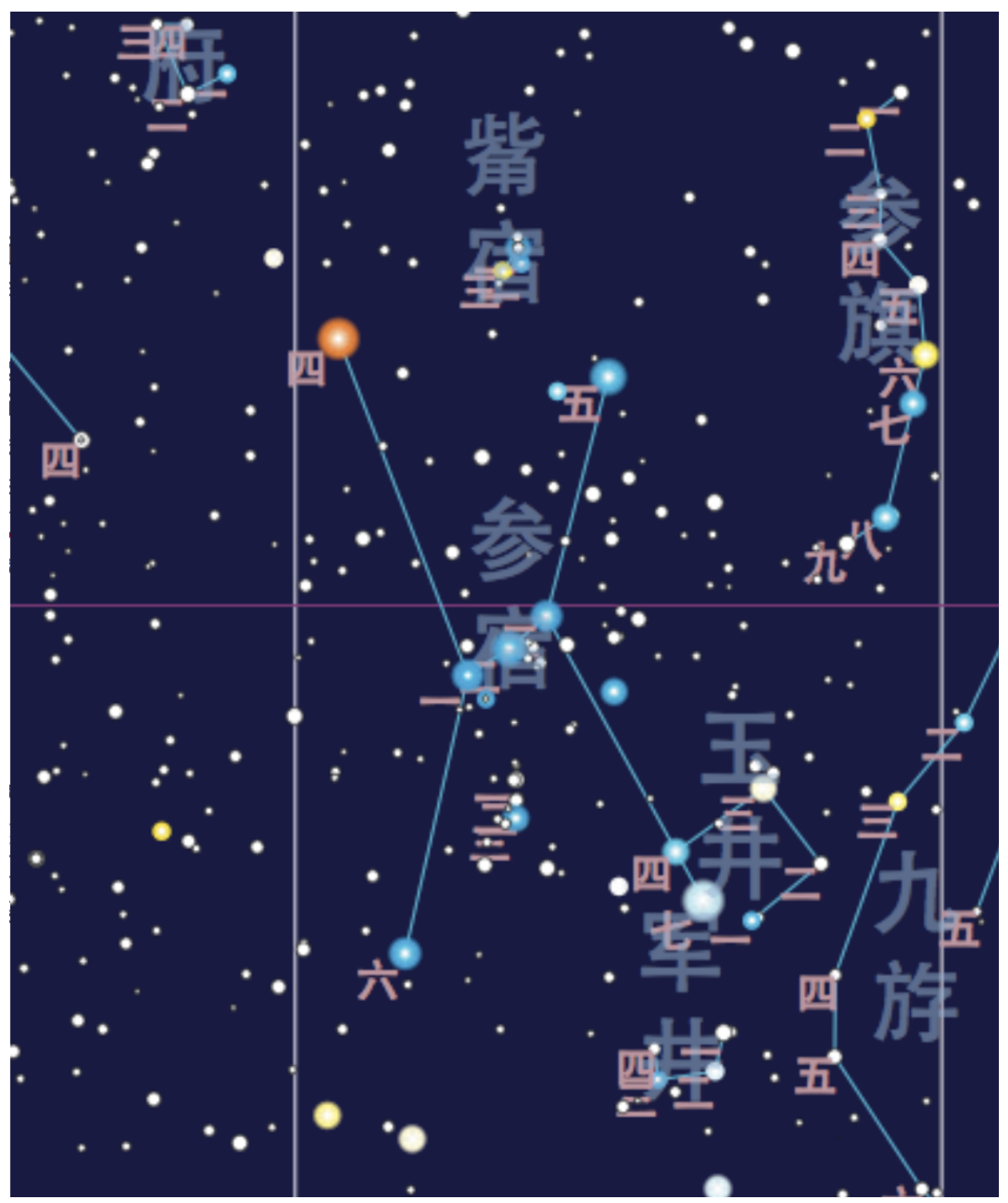

Figure 6: Image of the Three Stars mansion from the Chinese constellations (taken from Wikipedia).

[5] Ho, Peng Yoke, 1962, Vistas, 5, 127.

[6] Ho, Peng Yoke, 2003, Li, Qi and Shu, An Introduction to Science and Civilization in China, Chapter 5, Dover Science Books.

[7] Hsi, Tse-Tsung, 1958, Smithsonian Contr. Astrophys. 2, 109.

[8] Jacoby, G. H. et al 2010, PASA 27, 156.

[9] Johansson, G. H., 2007, JBAA 117, 5.

[10] Miszalski, B. et al, 2015. Work in progress.

[11] Nickiforov, M. G., 2010, Bulgarian AJ, 13, 116.

[12] Patterson, J. 2011, MNRAS 411, 2695.

[13] Patterson, J. et al, 2013, ApJ 434, 1902. 
[14] Shara, M. M. et al, 1986, ApJ 311, 163.

[15] Shara, M. M. et al, 2007, Nature 446, 159.

[16] Shara, M. M. et al, 2012a, ApJ 756, 107.

[17] Shara M. M. et al, 2012b, ApJ 758, 121.

[18] Warner, B., 1987, MNRAS 227, 23.

[19] Warner, B., 1995. Cataclysmic Variable Stars, CUP.

[20] Xu, Z., Pankanier, D. W., Jiang, Y., 2000, Culture and Cosmos, Vol 5, Gordon Breach 